\title{
An IC up-down counter controller for a programmable attenuator
}

\author{
LAWRENCE L. FETH and MICHAEL J. DONOHUE \\ Department of Audiology and Speech Sciences, Purdue University, West Lafayette, Indiana 47907
}

\begin{abstract}
A simple up-down counter is used to control a programmable attenuator. This controller reduces the load on a minicomputer or other solid state circuitry when the attenuator is used for adaptive testing in psychoacoustics. Circuit diagrams for the controller and an auxiliary display driver are given, and some applications are discussed.
\end{abstract}

Adaptive testing procedures have become very popular in psychoacoustic research. To implement many of these procedures, such as PEST (Taylor \& Creelman, 1967), the transformed up-down method (Levitt, 1971), or staircase procedures (Cornsweet, 1962), a variable attenuator is necessary. The variable attenuator, any one of several commercially available, is often programmable. That is, it is possible to move from one level of attenuation to any other level in one step, rather than the sequential movement required in older motordriven recording attenuators. The adaptive test procedures usually do not call for random access to attenuation levels. They are designed to move from one level to the next in either fixed or adjustable sized steps.

For psychoacoustics laboratories with modest minicomputer facilities, or in those with no computer at all, it may be difficult to dedicate the number of input/output lines required to allow fully random selection of attenuation level. We have designed and built a simple up-down counter which determines the attenuation level for our programmable attenuator. The minicomputer simply supplies either a "count-up" or a "count-down" pulse to the controller. Simple solid state logic circuits could also supply these control signals.

\section{CIRCUIT DESCRIPTION}

Our circuit has been designed to work with the Charybdis programmable attenuator. Other attenuators may require some slight modifications. Attenuation levels are set by raising individual input lines to a high logic level $(+2.0 \mathrm{~V} \mathrm{dc} \mathrm{min})$.

As supplied by Charybdis, attenuation values can be selected from 0 to $127.75 \mathrm{~dB}$ in $1 / 4-\mathrm{dB}$ steps. The attenuator has binary-coded control bits (i.e., 1/4-, 1/2-, 1-, 2-, 4-, 8-, 16-, 32-, and 64-dB control bits). For our use, we have not implemented the $1 / 4-$ and $1 / 2-\mathrm{dB}$ lines. (Note: Unused inputs on the Charybdis attenuator

This work was supported by a grant from the National Institutes of Health. should be grounded.) The attenuator input lines are connected to the output lines of the up-down counter.

Two 74193 IC chips are used to implement the updown counter. These up-down counters have separate up- and down-count lines as well as carry and borrow lines for cascading several stages. Figure 1 shows the schematic diagram. Attenuation Bits 1 through 8 are assigned to the first chip; 16 through 64 are set by the second.

The counter is preset to a selected value by a 7-bit switch register before the experimental run is begun. The eighth switch shown in Figure 1 is optional. The switch positions have no influences on the state of the counter under normal operating conditions. The counter can only be preset when the LOAD SWITCH REGISTER button is pushed.

The 74121 one-shot is used to deliver a load pulse to the attenuator 200 nanosec after the counter has been set. Each up-count or down-count pulse also activates the one-shot through the NAND gate so that the attenuation value is changed after each pulse. The other two gates on the 7410 are used as inverters. Computer lines are normally low and go high when the counter is to be changed.

The Charybdis attenuator provides output lines that are available to drive a display. Figure 2 shows the schematic of our decimal display circuit which converts the binary output of the attenuator into a $21 / 2$-digit decimal display. The output lines from the attenuator are delivered to a binary-to-BCD converter (three 74185 chips) which in turn drives a BCD seven-segment LED decoder/driver (the 7446 chips).

Before this display circuit was implemented, we simply mounted a LED above each SWITCH REGISTER position to indicate the status of the attenuator. These LEDs were connected directly to the attenuator output lines.

\section{APPLICATIONS}

We will describe several applications for which the up-down counter controller is suitable. Certainly, other adaptive testing procedures can be implemented in much the same manner. 


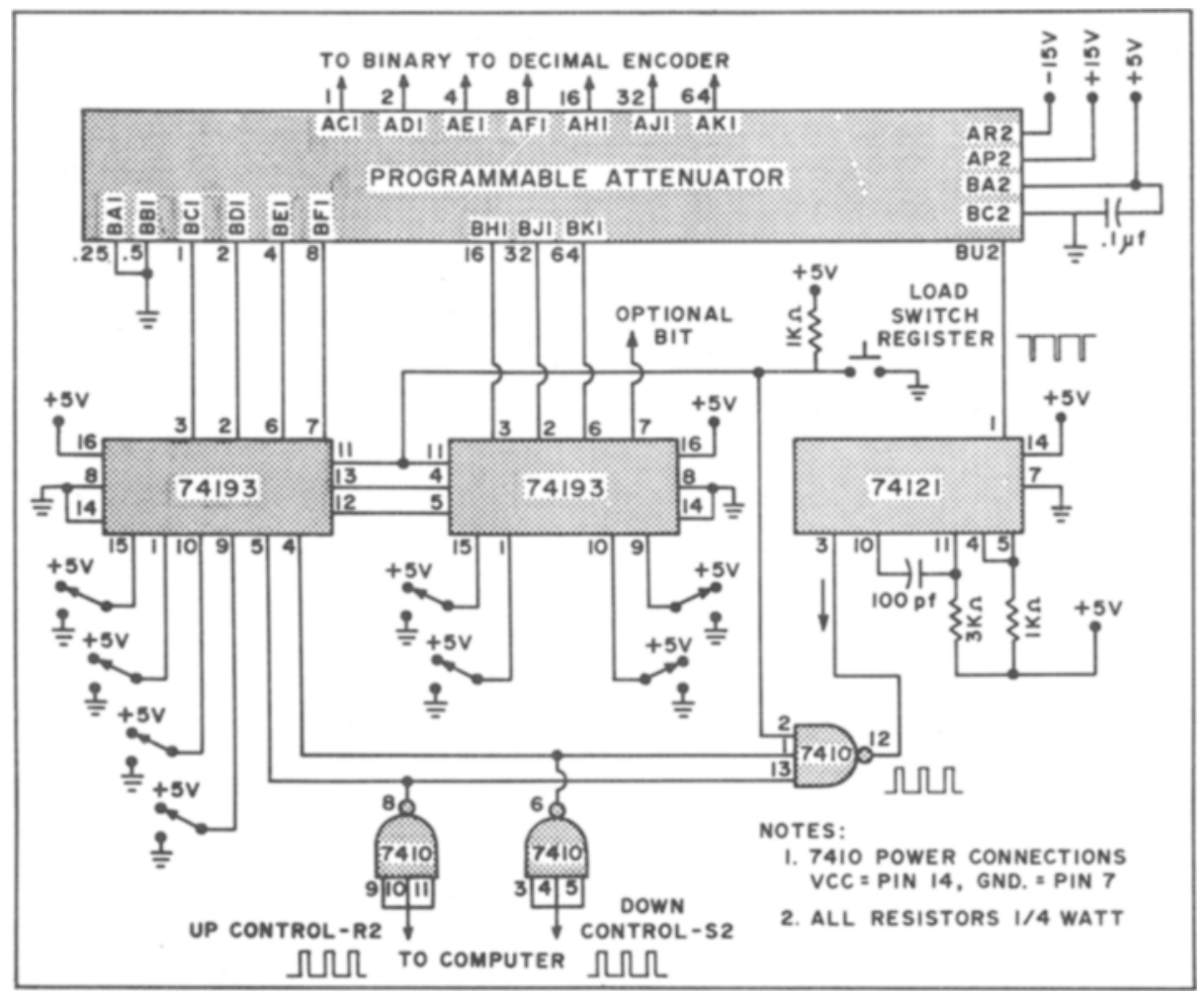

Figure 1. Schematic diagram of the up-down counter controller for a programmable attenuator. Control waveforms are illustrated at the control input points, the input to the one-shot and at the output of the one-shot. The arrow at the input to the one-shot indicates that the oneshot triggers on the falling edge of the waveform. The one-shot delay is set to approximately 200 nanosec.

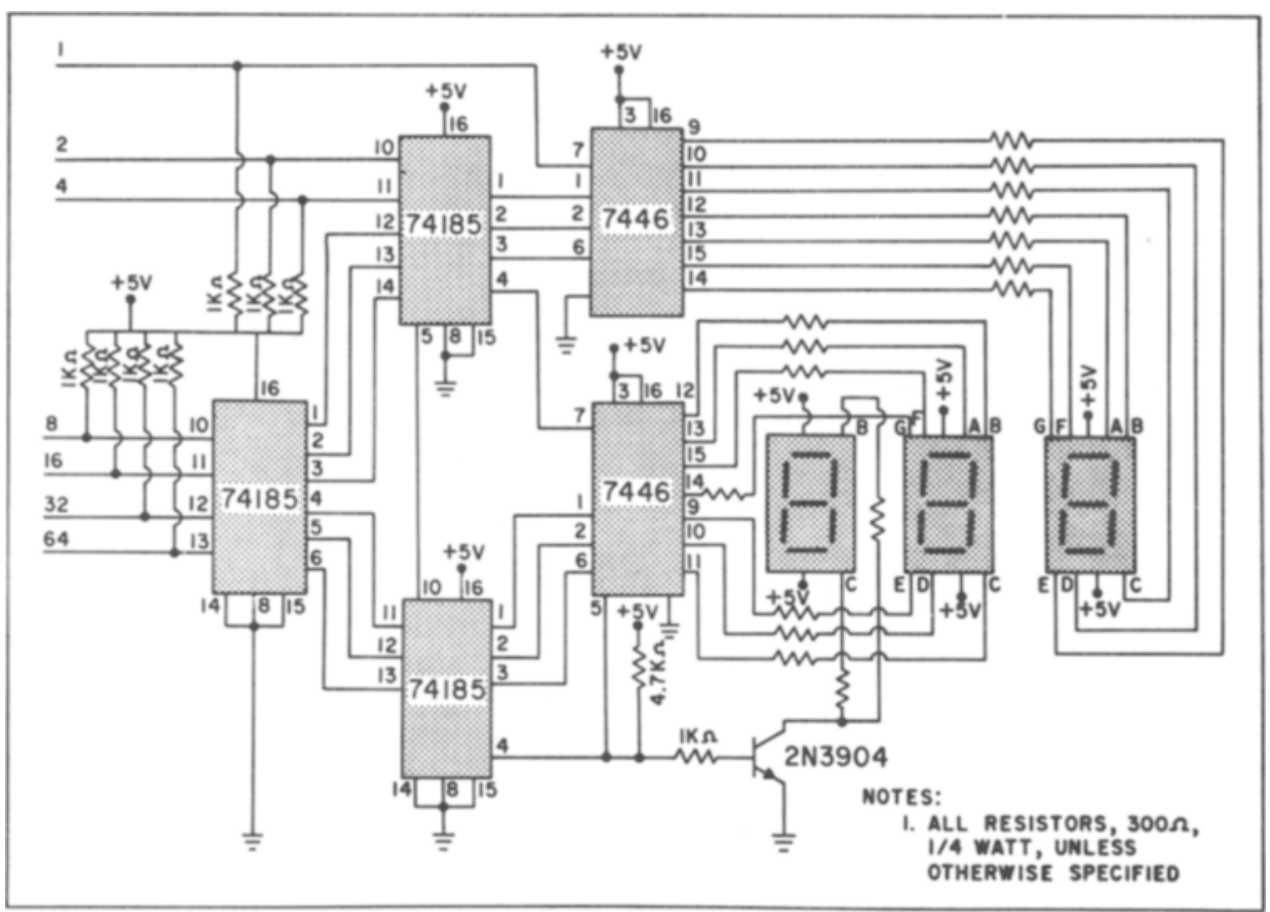

Figure 2. Schematic diagram of the optional decimal display. This circuit was designed specifically for the Charybdis attenuator. 
In the simplest use of the controller, we have implemented the tracking procedure which underlies the Békésy audiometric testing procedure. If, for example, an attenuation rate of $4 \mathrm{~dB} / \mathrm{sec}$ is desired, then every 250 msec the PDP-8/L computer sends a count-down pulse to the controller. The attenuation level falls at $4 \mathrm{~dB} / \mathrm{sec}$, thus increasing signal level by $4 \mathrm{~dB} / \mathrm{sec}$. $A$ buttonpushing response from the listener causes a jump to the control subprogram, which pulses the count-up line every $250 \mathrm{msec}$. Release of the pushbutton sends the program back into the count-down mode. Additional programming keeps track of starting level and the level and time of each reversal, and produces an average which is equivalent to visual interpolation of chart-paper tracings. The counter-controller and programmable attenuator are by no means the simplest or least expensive implementation of the tracking procedure. It is discussed here simply to illustrate the use of the system.

Forced-choice experiments are usually designed to have fixed time intervals for signal presentation, responses, and feedback. In a simple up-down forcedchoice procedure, the signal level is changed after every trial. If the previous response was correct, the level is lowered; if the subject was incorrect, the signal level is increased. Such a procedure can be implemented using this controller by presenting the control pulses during the warning or feedback intervals. In the more sophisticated procedures, signal level may not change after every presentation. Still, the programming of either a minicomputer or solid state logic can supply an appropriate count-up or count-down pulse at the correct time.
Thus, in one transformed up-down procedure (Levitt, 1971), the signal level is increased after every incorrect response and decreased only after two consecutive correct responses. Again, other programming records the starting level and total number of trials and determines the stopping point.

In the PEST procedure (Taylor \& Creelman, 1967), the size of a signal-level step varies with the past performance of the listener. The procedure begins by calling for an initial step size, then, as testing progresses, the step size may be halved or doubled. The counter controller is wired to produce a 1-dB change in level for each control pulse. For larger steps, the program simply delivers two, four, or more pulses to reach the new required level. If a step size smaller than $1 \mathrm{~dB}$ is desired, the counter can be wired to the attenuator in $1 / 2-\mathrm{dB}$, or even $1 / 4-d B$, steps. Of course, this then becomes the unit step size, so that an 8-dB step could require 16 or 32 pulses. These pulses can be delivered at a rate high enough to insure no hitch in most test procedures.

\section{REFERENCES}

Cornsweet, T. N. The staircase method in psychophysics. American Journal of Psychology, 1962, 75, 485-491.

LEviTT, H. Transformed up-down methods in psychoacoustics. Journal of the Acoustical Society of America, 1971. 49, 467.477.

Taylor, M. M., \& Creelman, C. D. PEST: Efficient estimates of probability functions. Journal of the Acoustical Society of America, 1967, 41, 782-787.

(Received for publication April 7. 1977; accepted May 7, 1977.) 